\title{
$J$-File Sum Rule Tests for the $3 s-3 p$ \\ Transition Array in Singly Ionized Nitrogen - Comparison of Measured and Calculated Data
}

\author{
J. MusieloK* \\ Institute of Physics, Opole University, Oleska 48, 45-052 Opole, Poland
}

(Received March 16, 2005; revised version May 18, 2005)

\begin{abstract}
A critical analysis of available experimental and theoretical data on transition probabilities for spectral lines of singly ionized nitrogen was performed. $J$-file sum rule tests for the $3 s-3 p$ triplet supermultiplet in NII were performed on the basis of recently calculated and measured line strengths. It is shown that the inclusion of intersystem transitions in the analysis leads to a better agreement of the data with the $J$-file sum rule prediction.
\end{abstract}

PACS numbers: 32.30.--r, 39.30.+w

\section{Introduction}

In recent years quite a large number of transition probabilities of spectral lines of light elements have been determined including data for singly ionized nitrogen $[1-7]$. Some of these experimental as well as theoretical studies provide also data for a few triplet-singlet intersystem transitions, being very interesting e.g. from astrophysical point of view. The energy level system of NII has a distinguished feature compared to the neighboring two members of the carbon isoelectronic sequence (CI, OIII) - the energy difference between the excited terms $3 s^{3} P^{\mathrm{o}}$ and $3 s^{1} P^{\mathrm{o}}$ is of the order of the fine structure splitting of the $3 s^{3} P^{\mathrm{o}}$ term $\left(\Delta E \approx 200 \mathrm{~cm}^{-1}\right)$ thus causing strong level mixing and consequently leading to rather strong triplet-singlet intersystem transitions.

*e-mail: musielok@uni.opole.pl 
In an old (1958) experimental paper by Mastrup and Wiese [8] a test of the $J$-file sum rule for two triplet supermultiplets $(3 s-3 p$ and $3 p-3 d)$ has been performed, showing noticeable departures from theoretical predictions. Due to the applied photographic data acquisition, much weaker intersystem lines have not been measured in that experiment. In the present paper the tests of the $J$-file sum rule for the $3 s-3 p$ supermultiplet are presented on a basis of recently measured and calculated data. The contributions from intersystem transitions have been included in the analysis.

\section{Analysis and reduction of the data}

In Fig. 1 the partial energy diagram for NII is shown with $15 L S$-allowed and $6 L S$-forbidden transitions being studied (the fine structure splitting is not shown in the figure). The number next to each arrow represents the number of spectral lines originating from transition between the two terms involved. As mentioned above the strong level mixing between the terms $3 s{ }^{3} P^{\circ}$ and $3 s{ }^{1} P^{\circ}$ causes intersystem transitions to the lower level $3 s^{1} P_{1}{ }^{\circ}$ originating from the three triplet terms: ${ }^{3} S_{1}$ (one line at $507.359 \mathrm{~nm}$ ), ${ }^{3} P_{1,2,3}$ (three lines at 465.453, 466.721, and $467.491 \mathrm{~nm}$ ), and ${ }^{3} D_{1,2}$ (two lines at 574.730 and $576.745 \mathrm{~nm}$ ).

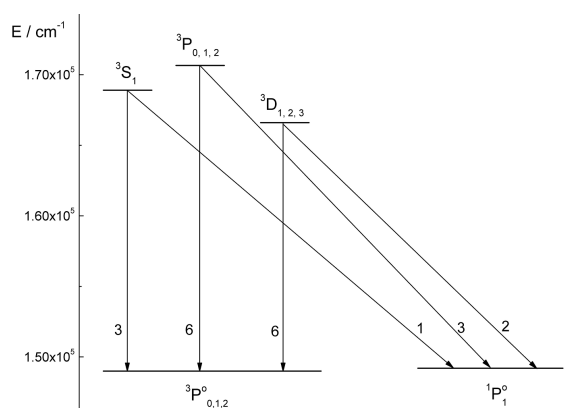

Fig. 1. Partial energy level diagram for singly ionized nitrogen is shown with the studied $L S$-allowed and the triplet-singlet intersystem transitions. The number nearby each transition represents the number of fine structure components originating from the two terms involved. The fine structure splitting into the respective energy levels is not shown.

A complete set of line strength data for transitions shown in Fig. 1 can be found in the experimental paper by Musielok et al. [2]. In that work transition probabilities have been determined by the emission method, applying a wall-stabilized arc as the excitation source. The measured total line intensities have been calibrated applying a tungsten strip standard lamp (calibrated by the Radiometric Division at the National Institute of Standards and Technology). The absolute scale for the transition probabilities was established by normalizing the 
data to the multiplet value of the $3 s{ }^{3} P^{\circ}-3 p{ }^{3} S$, taken from recently published NIST tables [9].

In the paper by Vaeck et al. [3] all data needed for the $J$-file rule test are listed except for one line at $567.956 \mathrm{~nm}$ (transition ${ }^{3} P_{2}^{\circ}-{ }^{3} D_{3}$ ). This missing value has been calculated using as a reference the $A_{k i}$ value for the transition ${ }^{3} P_{1}^{\circ}-{ }^{3} D_{2}$ taken from a former paper published by this group (Bell et al. [1]), assuming that the ratio of transition probabilities $A\left({ }^{3} P_{2}^{\circ}-{ }^{3} D_{3}\right) / A\left({ }^{3} P_{1}{ }^{\circ}-{ }^{3} D_{2}\right)$ calculated in [1] should be preserved in the more recent calculation of Vaeck et al. The following formula has been used to calculate the transition probability for the ${ }^{3} P_{2}{ }^{\circ}-{ }^{3} D_{3}$ fine structure component:

$$
\begin{aligned}
& A\left({ }^{3} P_{2}^{\text {o }}-{ }^{3} D_{3}\right)^{\text {Ref. }}{ }^{[3]}= \\
& \quad A\left({ }^{3} P_{1}^{\text {o }}-{ }^{3} D_{2}\right)^{\text {Ref. }[}\left[{ }^{[3]}\left[A\left({ }^{3} P_{2}^{\text {o }}-{ }^{3} D_{3}\right) / A\left({ }^{3} P_{1}^{\text {o }}-{ }^{3} D_{2}\right)\right]^{\text {Ref. }}{ }^{[1]} .\right.
\end{aligned}
$$

For the sake of the present analysis the transition probabilities listed in [3], determined within the "length" and "velocity" approximation were averaged (arithmetic mean).

In a recent paper by Lavin et al. [7] transition probabilities have been calculated applying the relativistic quantum defect orbital (RQDO) method. Since these calculations have been performed applying the $L S$ coupling scheme, only $L S$-allowed data are reported. The paper provides two data sets: (i) without and (ii) with explicit polarization correction. For our analysis the latter set of results (determined with explicit polarization correction) have been used. In Table 6 of Ref. [7] the listed transition probability values for the spectral line at $573.066 \mathrm{~nm}$ are charged with an obvious printing error - the quoted results (even those taken from other papers) should be one order of magnitude smaller. For the present analysis this necessary correction has been performed.

In addition, two other experimental data sets have been taken for comparison with measured and calculated $A_{k i}$ values.

The paper by Mastrup and Wiese [8] mentioned already in Introduction provides a complete set of line strengths for all $L S$-allowed transitions for the triplet supermultiplet. In a recent work by Mar et al. [6] besides of $L S$-allowed transitions, data for two singlet-triplet intersystem transitions are reported. However, because of some experimental difficulties, results for two rather strong $L S$-allowed transitions of the ${ }^{3} P^{\circ}-{ }^{3} S$ multiplet $(\lambda=501.062$ and $500.270 \mathrm{~nm})$ are missing in this paper.

The selected experimental and theoretical data sets (transition probabilities) have been transformed into line strength units, applying the formula [10]:

$$
S_{i k}=4.9356 \times 10^{-19} \lambda^{3} g_{k} A_{k i},
$$

where $g_{k}$ is the statistical weight of the upper level. The line strengths $S_{i k}$ are obtained in the so-called atomic units, the transition probabilities $A_{k i}$ have to be taken in units $\left(s^{-1}\right)$ and the wavelengths $\lambda$ in angstroms. 
In Figs. 2 and 3 experimental $A_{k i}$ values taken from Refs. [2, 6] and [8] are compared with results calculated by Vaeck et al. [3]. In Fig. 2 the ratios

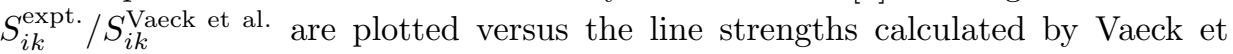

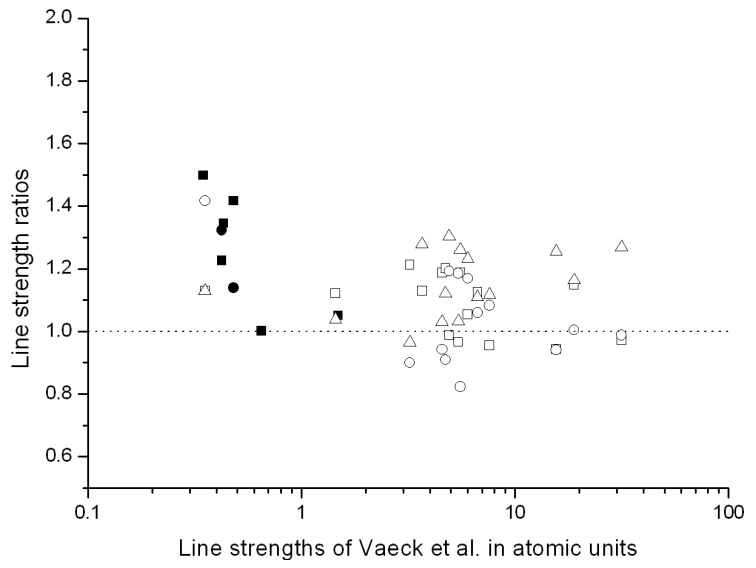

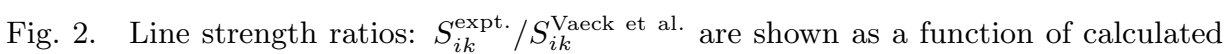
line strengths by Vaeck et al. [3]. The open symbols represent ratios of $L S$-allowed transitions, while the solid ones those of intersystem transitions. Squares (口匹) represent the $S_{i k}$ ratios of Ref. [2]/Ref. [3]; triangles $(\triangle)$ the ratios of Ref. [8]/Ref. [3] and circles (॰•) the ratios of Ref. [6]/Ref. [3].

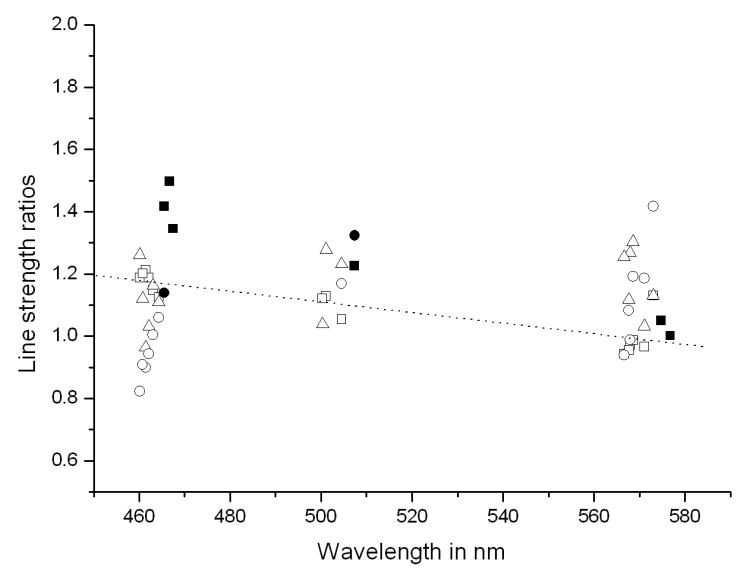

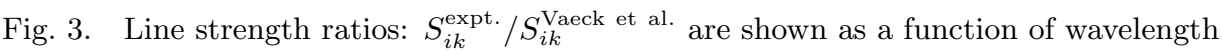
of respective spectral lines. The open symbols represent ratios of $L S$-allowed transitions, while the solid ones those of intersystem transitions. Squares (ם) represent the $S_{i k}$ ratios of Ref. [2]/Ref. [3]; triangles $(\triangle)$ the ratios of Ref. [8]/Ref. [3] and circles (०•) the ratios of Ref. [6]/Ref. [3]. The straight line represents the best fit to the data when $L S$-allowed line strengths of Ref. [2] and Ref. [3] are compared. 
al. [3], while in Fig. 3 the same ratios are shown as a function of wavelengths. In Fig. 2 a typical scatter of the data can be seen. Some of the discrepancies between calculations and the measured data originate from the determination of the absolute scale of the experimental results. A lack of noticeable dependence of the line strength ratios on calculated line strengths can be regarded as a proof that the measured data have not been charged with errors, originating from possible departure of the respective light sources from optically thin conditions.

Nearly a similar scatter is visible in Fig. 3, apart from a rather clear trend of the data by Musielok et al. [2] (squares). The straight line in this figure represents a best fit to the results determined for $L S$-allowed transitions (open squares). The same trend can also be seen in the case of the intersystem lines (solid squares). The agreement at wavelengths around $570 \mathrm{~nm}\left({ }^{3} \mathrm{P}^{\mathrm{o}}-{ }^{3} \mathrm{D}\right.$ transitions) is excellent but at wavelengths about $460 \mathrm{~nm}\left({ }^{3} P^{\circ}-{ }^{3} P\right.$ transitions $)$ the measured line strengths are about $20 \%$ larger than the calculated data. It seems that the measurements of Ref. [2] are charged with a noticeable systematic error, originating probably from the calibration procedure against the signals determined from the radiometric standard source.

\section{3. $J$-file sum rule tests}

The $J$-file sum rule tests have been performed in two steps. In the first step only $L S$-allowed transitions have been taken into account. The line strengths for all allowed transitions taken from Ref. [3] have been added in order to obtain the total strength of the $3 s-3 p$ triplet supermultiplet $\left(S_{\text {allowed }}=120.16\right.$ a.u.). The two other data sets taken for comparison: the theoretical data of Lavin et al. [7] and the experimental data of Ref. [2] have been then normalized to the total supermultiplet strength obtained for the data of Vaeck et al. [3]. The results are presented in Table I. In the last column and the three last lines of Table I the discrepancies between the analyzed data and the $J$-file sum rule are quoted (in percent). For all three analyzed data sets, the sum of line strengths for transitions involving the lower level ${ }^{3} P_{1}^{\circ}$ is smaller, while for transitions involving the levels ${ }^{3} P_{0}^{\circ}$ and ${ }^{3} P_{2}^{\circ}$ larger than expected from the $J$-file sum rule. Noticeable discrepancies are also encountered when the sums of line strengths summed up for transitions originating from different upper terms but for levels with given $J$-values.

In the second step line strengths for intersystem lines have been included in the analysis. For this test only two data sets are available. Similarly, the experimental data of Ref. [2] have been normalized to the total strength of the supermultiplet determined for the data of Vaeck et al. including the line strengths for intersystem transitions $\left(S_{\text {all. }+ \text { forb. }}=124.0\right.$ a.u. $)$. The results are presented in Table II. As can be seen, the results for all lower levels of the term ${ }^{3} P^{\circ}$, the inclusion of intersystem transitions reduces significantly the discrepancies observed in the case of the former analysis (see Table I), leading to an almost perfect agreement with the $J$-file rule prediction. 
TABLE I

$J$-file sum rule test for the $3 s-3 p$ transition array of NII (triplet supermultiplet, $L S$-allowed transitions). The results given in italics are calculated data: in the upper line of Vaeck et al. [3], in the middle line of Lavin et al. [7]; those given in regular characters represent the experimental data of Musielok et al. [2].

\begin{tabular}{|c|c|c|c|c|c|c|c|c|c|}
\hline $\begin{array}{l}3 p \\
3 s\end{array}$ & ${ }^{3} P_{0}$ & ${ }^{3} S_{1}$ & ${ }^{3} P_{1}$ & ${ }^{3} D_{1}$ & ${ }^{3} P_{2} \quad{ }^{3} D_{2}$ & ${ }^{3} D_{3}$ & $\sum$ & $\begin{array}{c}J \text {-file } \\
\text { rule }\end{array}$ & $\begin{array}{c}\text { Discre- } \\
\text { pancy } \%\end{array}$ \\
\hline${ }^{3} P_{0}^{\mathrm{o}}$ & - & $\begin{array}{l}1.44 \\
1.46 \\
1.54 \\
\end{array}$ & $\begin{array}{l}4.73 \\
4.62 \\
5.46 \\
\end{array}$ & $\begin{array}{l}7.60 \\
7.34 \\
6.92 \\
\end{array}$ & - & - & $\begin{array}{l}13.77 \\
13.42 \\
13.88\end{array}$ & 13.35 & $\begin{array}{l}+3.1 \\
+0.5 \\
+4.0\end{array}$ \\
\hline${ }^{3} P_{1}^{\circ}$ & $\begin{array}{l}4.55 \\
4.62 \\
5.16\end{array}$ & $\begin{array}{l}3.67 \\
4.38 \\
3.96\end{array}$ & $\begin{array}{l}3.22 \\
3.24 \\
3.72\end{array}$ & $\begin{array}{l}4.91 \\
5.51 \\
4.62\end{array}$ & $\begin{array}{ll}5.55 & 15.61 \\
5.41 & 16.53 \\
6.29 & 14.05 \\
\end{array}$ & - & $\begin{array}{l}37.51 \\
35.07 \\
37.80\end{array}$ & 40.06 & $\begin{array}{l}-6.4 \\
-2.5 \\
-5.6\end{array}$ \\
\hline${ }^{3} P_{2}{ }^{\circ}$ & - & $\begin{array}{l}6.00 \\
7.31 \\
6.05\end{array}$ & $\begin{array}{l}6.67 \\
5.80 \\
7.16\end{array}$ & $\begin{array}{l}0.35 \\
0.37 \\
0.38\end{array}$ & $\begin{array}{ll}18.91 & 5.43 \\
17.39 & 5.50 \\
20.69 & 4.99\end{array}$ & $\begin{array}{l}31.52 \\
30.68 \\
29.21\end{array}$ & $\begin{array}{l}68.88 \\
67.05 \\
68.48\end{array}$ & 66.75 & $\begin{array}{l}+3.2 \\
+0.5 \\
+2.6\end{array}$ \\
\hline$\sum$ & $\begin{array}{l}4.55 \\
4.62 \\
5.16 \\
\end{array}$ & $\begin{array}{l}11.11 \\
13.15 \\
11.55 \\
\end{array}$ & $\begin{array}{l}14.62 \\
13.66 \\
16.30\end{array}$ & $\begin{array}{l}12.86 \\
13.22 \\
11.92 \\
\end{array}$ & $\begin{array}{l}24.4621 .04 \\
22.8022 .03 \\
26.9819 .04\end{array}$ & $\begin{array}{l}31.52 \\
30.68 \\
29.21 \\
\end{array}$ & $\begin{array}{l}120.16 \\
120.16 \\
120.16 \\
\end{array}$ & & 120.16 \\
\hline $\begin{array}{l}\text { Mean } \\
\text { value } \\
\text { for } J\end{array}$ & $\begin{array}{l}4.55 \\
4.62 \\
5.16\end{array}$ & & $\begin{array}{l}12.86 \\
13.34 \\
13.26\end{array}$ & & $\begin{array}{l}22.75 \\
22.41 \\
23.01\end{array}$ & $\begin{array}{l}31.52 \\
30.68 \\
29.21\end{array}$ & - & & - \\
\hline $\begin{array}{c}J \text {-file } \\
\text { rule }\end{array}$ & $\begin{array}{c}4.45 \\
\times 1 \\
\end{array}$ & & $\begin{array}{c}13.35 \\
\times 3\end{array}$ & & $\begin{array}{c}22.25 \\
\times 2\end{array}$ & $\begin{array}{c}31.16 \\
\times 1\end{array}$ & - & & 120.16 \\
\hline $\begin{array}{c}\text { Dis- } \\
\text { cre- } \\
\text { pancy } \\
\%\end{array}$ & $\begin{array}{l}+2.2 \\
+3.0 \\
+16.0\end{array}$ & & $\begin{array}{l}-3.7 \\
<-0.1 \\
-0.7\end{array}$ & & $\begin{array}{l}+2.2 \\
+0.3 \\
+3.4\end{array}$ & $\begin{array}{l}+1.1 \\
-1.5 \\
-6.2\end{array}$ & - & & - \\
\hline
\end{tabular}

However, the discrepancies observed for the upper levels become even larger, especially in the case of the experimental data set. The reason for this rather large departure of the experimental data for the levels ${ }^{3} P_{0}(+24 \%)$ and ${ }^{3} D_{3}(-9.5 \%)$ is probably caused by the formerly mentioned possible error in the determination of absolute transition probabilities (see the best fit straight line in Fig. 3). Indeed, from this figure one has to expect that the line strengths for transitions with shorter wavelengths around $460 \mathrm{~nm}$ (second column in Table II, transitions ${ }^{3} P_{1}^{\mathrm{o}}-{ }^{3} P_{0}$ and $\left.{ }^{1} P_{1}^{\circ}-{ }^{3} P_{0}\right)$ are overestimated and consequently the line strengths for transition with longer wavelengths around $570 \mathrm{~nm}$ (fifth column, spectral line ${ }^{3} P_{2}{ }^{\mathrm{o}}-{ }^{3} D_{3}$ ) have to be expected to be underestimated. 
TABLE II

$J$-file sum rule test for the $3 s-3 p$ transition array of NII (triplet supermultiplet, including triplet-singlet intersystem transitions). The results given in italics are calculated data of Vaeck et al. [3], those given in regular characters represent the experimental data of Musielok et al. [2].

\begin{tabular}{|c|c|c|c|c|c|c|c|c|c|}
\hline $3^{3 p}$ & ${ }^{3} P_{0}$ & ${ }^{3} S_{1}$ & ${ }^{3} P_{1}$ & ${ }^{3} D_{1}$ & ${ }^{3} P_{2} \quad{ }^{3} D_{2}$ & ${ }^{3} D_{3}$ & $\sum$ & $\begin{array}{c}J \text {-file } \\
\text { rule }\end{array}$ & $\begin{array}{c}\text { Discre- } \\
\text { pancy \% }\end{array}$ \\
\hline${ }^{3} P_{0}^{o}$ & - & $\begin{array}{l}1.44 \\
1.54 \\
\end{array}$ & $\begin{array}{l}4.73 \\
5.40 \\
\end{array}$ & $\begin{array}{l}7.60 \\
6.90 \\
\end{array}$ & - & - & $\begin{array}{l}13.77 \\
13.84 \\
\end{array}$ & 13.78 & $\begin{array}{l}<-0.1 \\
+4.0\end{array}$ \\
\hline${ }^{3} P_{1}^{\circ}$ & 4.55 & 3.67 & 73.22 & 4.91 & 5.5515 .61 & & 37.51 & & $<+0.1$ \\
\hline${ }^{1} P_{1}^{\circ}$ & 0.44 & 0.43 & 0.35 & 0.65 & $0.48 \quad 1.49$ & - & +3.84 & $\begin{array}{l}41.35 \\
41.33\end{array}$ & \\
\hline${ }^{3} P_{1}^{\mathrm{o}}$ & 5.14 & 3.94 & 3.70 & 4.60 & 6.2614 .00 & & 37.64 & 41.93 & \\
\hline${ }^{1} P_{1}^{\mathrm{o}}$ & 0.56 & 0.49 & 0.49 & 0.62 & $0.65 \quad 1.48$ & & +4.29 & & +1.0 \\
\hline${ }^{3} P_{2}^{o}$ & - & $\begin{array}{l}6.00 \\
6.02\end{array}$ & $\begin{array}{l}6.67 \\
7.14\end{array}$ & $\begin{array}{l}0.35 \\
0.38\end{array}$ & $\begin{array}{l}18.91 \quad 5.43 \\
20.624 .97\end{array}$ & $\begin{array}{l}31.52 \\
29.10\end{array}$ & $\begin{array}{l}68.88 \\
68.23\end{array}$ & 68.89 & $\begin{array}{c}<-0.1 \\
-0.6\end{array}$ \\
\hline$\sum$ & $\begin{array}{l}4.99 \\
5.70 \\
\end{array}$ & $\begin{array}{l}11.54 \\
11.90 \\
\end{array}$ & $\begin{array}{l}14.97 \\
16.73\end{array}$ & $\begin{array}{l}13.51 \\
12.50 \\
\end{array}$ & $\begin{array}{l}24.9422 .53 \\
26.9819 .04\end{array}$ & $\begin{array}{l}31.52 \\
29.10 \\
\end{array}$ & $\begin{array}{l}124.0 \\
124.0\end{array}$ & & 124.0 \\
\hline $\begin{array}{l}\text { Mean } \\
\text { value } \\
\text { for } J\end{array}$ & $\begin{array}{l}4.99 \\
5.70\end{array}$ & & $\begin{array}{l}13.33 \\
13.74 \\
\end{array}$ & & $\begin{array}{l}23.74 \\
23.99 \\
\end{array}$ & $\begin{array}{l}31.52 \\
29.10 \\
\end{array}$ & - & & - \\
\hline $\begin{array}{l}J \text {-file } \\
\text { rule }\end{array}$ & $\begin{array}{c}4.59 \\
\times 1 \\
\end{array}$ & & $\begin{array}{c}13.78 \\
\times 3 \\
\end{array}$ & & $\begin{array}{c}22.96 \\
\times 2 \\
\end{array}$ & $\begin{array}{c}32.15 \\
\times 1 \\
\end{array}$ & - & & 124.0 \\
\hline $\begin{array}{c}\text { Dis- } \\
\text { cre- } \\
\text { pancy } \\
\%\end{array}$ & $\begin{array}{r}+8.7 \\
+24.0\end{array}$ & & $\begin{array}{c}<-3.2 \\
-0.3\end{array}$ & & $\begin{array}{l}+3.4 \\
+4.4\end{array}$ & $\begin{array}{l}-2.0 \\
-9.5\end{array}$ & - & & - \\
\hline
\end{tabular}

\section{Conclusions}

The analysis of line strength data of singly ionized nitrogen available in literature and particularly the tests of the $J$-file sum rule for the $3 s-3 p$ supermultiplet performed in this work, lead to the following conclusions:

- the experimental results obtained in [2] are affected by a systematic error influencing also the results of branching ratio measurements;

- the inclusion of $L S$-forbidden transitions leads to a much better agreement with the $J$-file sum rule prediction; 
— the set of line strengths based on recent CIV3 calculations [3] (and [1]) presented in Table II fulfills the $J$-file sum rule with good accuracy and is recommended for further use e.g. for plasma diagnostics purposes.

\section{References}

[1] K.L. Bell, A. Hibbert, R.P. Stafford, Phys. Scr. 52, 240 (1995).

[2] J. Musielok, J.M. Bridges, S. Djurović, W.L. Wiese, Phys. Rev. A 53, 3122 (1996).

[3] N. Vaeck, J. Fleming, K.L. Bell, A. Hibbert, M.R. Godefroid, Phys. Scr. 56, 603 (1997)

[4] Ch. Froese-Fischer, Phys. Scr. Vol. T 83, 49 (1999).

[5] N. Zheng, T. Wang, R. Yang, Y. Wu, J. Chem. Phys. 112, 7042 (2000).

[6] S. Mar, C. Perez, V.R. González, M.A. Gigosos, J.A. del Val, I. de la Rosa, J.A. Aparicio, Astron. Astrophys. Suppl. Ser. 114, 509 (2000).

[7] C. Lavin, E. Olalla, I. Martin, Astrophys. J. 543, 1051 (2000).

[8] F. Mastrup, W.L. Wiese, Z. Astrophys. 44, 259 (1958).

[9] W.L. Wiese, J. Fuhr, T.M. Deters, Atomic Transition Probabilities for Carbon, Nitrogen, and Oxygen, J. Phys. Chem. Ref. Data, Monograph No. 7, AIP, New York 1995.

[10] W.C. Martin, W.L. Wiese, in: Atomic, Molecular and Optical Physics Handbook, Ed. G.W.F. Drake, American Institute of Physics, Woodbury 1996, p. 135. 